\title{
Computational Fluid Dynamics Investigation of Counterflow Ejection Effects on a Body moving with High Speed
}

\author{
Y.Y Zheng \\ Aerospace Engineering \\ School of Mechanical and Manufacturing Engineering, \\ University of New South Wales, NSW, Australia \\ y.y.zheng@unsw.edu.au
}

\author{
N.A.Ahmed \\ Aerospace Engineering \\ School of Mechanical and Manufacturing Engineering, \\ University of New South Wales, NSW, Australia
}

\begin{abstract}
A major problem associated with the process of slowing down a body moving with high speed, such as during reentry of a space craft, is the extreme heat generated. Sink and ablation cooling are commonly used but the cost of such heat protection systems is very high. Thus heat dissipation has been an area of extensive research. The simulation work presented in this paper is attempted to replenish flow/ thermal field reaction with a greater details of the flow field affected by the deployment of counterflow jet ejection in supersonic freestream and test the suitability of different turbulence models. An Apollo command re-entry module has been employed and it has been subjected to a free stream Mach number of 3.0. ANSYS, as the computational fluid dynamics tool has been performed in this two-dimensional numerical flow simulation. Effects of counterflow jet injection effects and its interaction with the oncoming flow through the frontal stagnation point of the test module are discussed. Results obtained from different turbulence models are compared and analyzed. The overall outcomes are highly promising. Within those turbulence models, shear stress transport model (SST) seems to be preferred for such simulation. It also appears that substantial heat reduction on the body is possible using appropriate strength counter flow jet.
\end{abstract}

\section{Keywords-counterflow jet ejection, heat, turbulence model}

\section{INTRODUCTION}

Since the end of the second world war, there has been continued interest in the application of active flow control concepts to modify or change the external flow fields of vehicles in order to reduce wave drag and aero-thermal loads[1]. The Aerodynamics Research team at the University of New South Wales has been developing various flow diagnostic methodologies[2-15] and active flow control techniques[16-31] for a multitude of applications over the years of which deploying counter flow jet appears to be a promising solution to the above problem, and is explored further in this paper.

After a review of numerical simulation of counter flow jet, some methods used by other researchers are summarized: Kentaro et al[32] use axisymmetric full Navier-Stokes equations as governing equations. Central difference is used for viscous terms. For the turbulence model, k- $\varepsilon$ is used. Free stream conditions are set equal to average conditions of experiment: $\mathrm{M}=3.98, \mathrm{P}=1.37 \mathrm{MPa}, \mathrm{T}=397 \mathrm{~K}$. For the boundary conditions, the pressure gradient perpendicular to the wall is assumed to be zero and no slip boundary condition is applied on the wall. Numerical results show that the recirculation region plays an important role for the reduction of heat flux. For the reduction of the aerodynamic heating, it is effective to cover the body surface with the cool jet flow to form strong circulation region.

Olekandr et al[33] set the 2-D model with the jet. The mesh used in computation consisted of 13,372 nodes after systematic grid refinement study when further refinement produced insignificant change in the results. Simulations are done using both $\mathrm{k}-\varepsilon$ and shear stress transport (SST) models to compare. The design Mach number is 1.50. The liner system of equations for mass, momentum, and energy is solved simultaneously using the implicit coupled solver iteratively by a block Gauss-Seidel method. The near flow field interaction under supersonic conditions indicates that the presence of small jet may alter the large supersonic flow field by altering the localized flow field pressure and weakening the strength of the shock waves. Range of minor jet total pressures were observed to eliminate the conical shock wave structure for the main jets, while allowing the minor jet flow to penetrate deeper into the main jet.

When it comes to Daso's[33] work, their work involved the use of a 3-dimensional structured-grid Renolds-averaged Navior-Stokes equations solver, with perfect gas, finite-rate chemistry and equilibrium air curve fit options, and has been widely used and validated. The turbulence model is the point wise Glodberg one equation turbulence model. The computational grids were generated with HyPerComp's TEMPUS-GRID structured and unstructured grid generator. The computational gird includes 750,000 points to nodes in total. The solutions were obtained with the perfect gas option of the solver and Goldberg's one equation turbulence model. In terms of heat transfer, the results show significant reduction in heat flux even giving negative heat flux for most of the short penetration mode interactions. Their findings strongly suggest that the application of counterflow jets as active flow control

The authors acknowledge Prof. Wei-Hua Zhang from National University of Defense Technology of China for his helpful comments on this project. 
could have strong impact on supersonic and hypersonic vehicle design and performance.

This paper, therefore, is an attempt to replenish flow/ thermal field reaction with a greater detail of the flow field affected by the deployment of counterflow jet ejection under supersonic freestream using numerical simulation. Different turbulence model will be used; effect of jet and turbulence model selection will be discussed further.

\section{NUNMERICAL SIMULATION}

\section{A. Numerical Model}

2-D Apollo Re-entry Model is selected. The angle of attack of re-entry process is settled as zero degrees with a velocity of Mach 3.0. Simulations are accomplished under ANSYS-CFX.

The schematic diagram is shown in Figure. 1 below. On this state, only 2-D case is cared, so symmetry plane will be used. As illustrated in figure, computational domain consisted of inlet of fluid domain, outlet of fluid domain, wall of Apollo body, two symmetric faces and opening side walls.

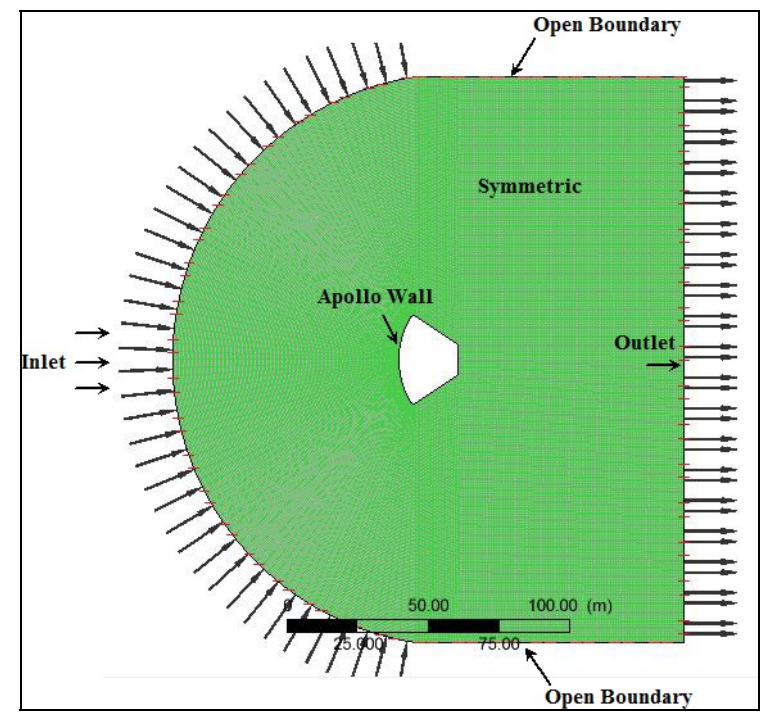

Figure 1. Schematic of the FlowDomain

In this study, because of the exploratory nature of the work, the quick qualitative analysis of the flow field due to the counterflow jet configuration mentioned earlier was desired rather than quantitatively accurate solution, the governing equations for momentum and energy were discretized with a conservative finite-volume formulation using only first order.

For the simulation of counterflow ejection, a jet is settled later on at the stagnation point. The function of jet simulation is realized through adding an extra inlet boundary condition. The geometry data used comes from references, and the dimension of jet is taken from real experiment. Obviously, the flow is expected to be turbulent, shock wave oscillation and flow instability will exist as a reaction to the jet performance. For the observation of the effect of different turbulence model usage, several turbulence models such as k- $\varepsilon$, k-Omega, SST and RSL Reynolds Stress model were employed under the same conditions.

\section{B. Grid Description}

The structured mesh is built under ANSYS-ICEM. This mesh consists of 98,299 elements and 64,800 nodes. It was chosen after systematic grid refinement study which involved the addition of more elements along the original inlet flow domain, namely, 77,089 elements and 50,720 nodes in total, until the refinement produced insignificant change in the results. Figure. 2 gives an overview of mesh details around the Apollo body and counterflow jet. The unit of dimension is millimeter.

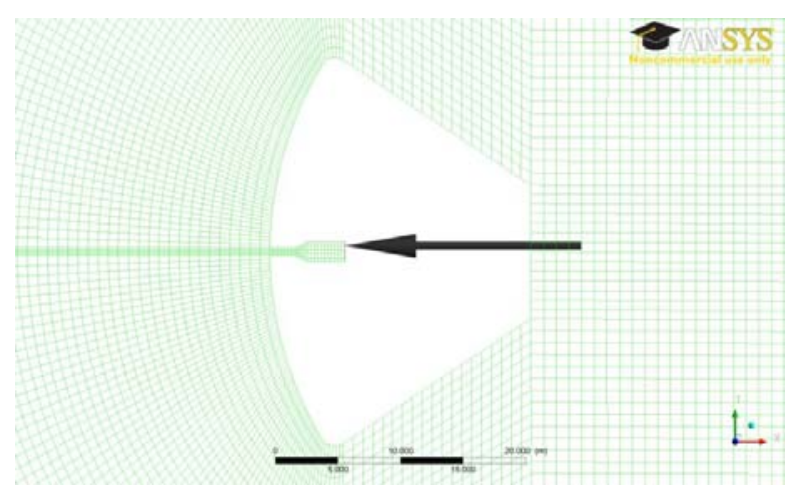

Figure 2. Mesh Details with Counterflow Jet

\section{Boundary Conditions}

In the pursuit of setting proper physical boundary conditions such as in the section of local refinement and solution adaptation are governed by the computational stability as well as the numerical convergence of the CFD problem.

In this project, one of the most important issues was the jet boundary. From the previous simulation experience, the jet boundary is better settled as opening boundary with input of Cartesian velocity. Because when jet boundary is chosen as inlet boundary, the results indicated that countercurrent flow will flow through the jet, which is not expected and reliable. Therefore, opening boundary is better described by observing the flow movement patterns.

Another part is the setting of wall boundary. For the two side edges (up and down) of fluid domain, it could be settled as either free slip wall or opening boundary. With regard to that when performing physical experiment, the test region in the wind tunnel formed into a closed area, the free slip wall is suggested as is it stands closer to experimental conditions.

\section{Turbulence Modelling}

Computation of the Reynolds stresses and turbulent heat fluxes for the closure of the governing equations requires turbulence modeling.

Keysar and Degani[34] used the Fluent CFD code and the $\mathrm{k}-\varepsilon$ turbulence model[35] and it variants such as the RNG k- $\varepsilon$ model develop by Yaknot et al[36] and the realizable k- $\varepsilon$ model developed by Shi et al[37] for the computational of fully expanded as well as under/over expanded axisymmetric turbulent jets. Additionally, they used the non-isotropic fourequation Reynolds stress turbulence model of Launder et al[38] for the computation. All characteristic flow features were 
correctly resolved, and good quantitative agreement with experimental data was achieved in their computation.

For the numerical study of counterflow jet, in order to compare the function of different turbulence models, firstly, laminar model (no turbulence) is introduced (see Figure.3) then the shear stress transport (SST) turbulence model, k- $\varepsilon$ turbulence model, k-Omega turbulence model, and RSL Reynolds Stress turbulence model are employed.

It shall be mentioned that the governing equations for conservation of mass, momentum, and turbulent properties are integrated over the mesh cells to yield a set of linear algebraic equations. The medium of fluid was chosen as air ideal gas. The turbulence options are all selected as medium. Other basic settings included the velocity of upstream flow (Mach 3.0), default temperature $(300 \mathrm{~K})$ and angle of attack ( 0 degree).

\section{RESULTS AND DISCUSSIONS}

Preliminary study shows that with all the results, SST model and k-Omega model are the most suitable ones for solving this problem. The two models convergence quickly and can obtain a smooth solution within the discontinuous part. The distance between the bow shock wave and the stagnation point on the body is marked by an arrow.
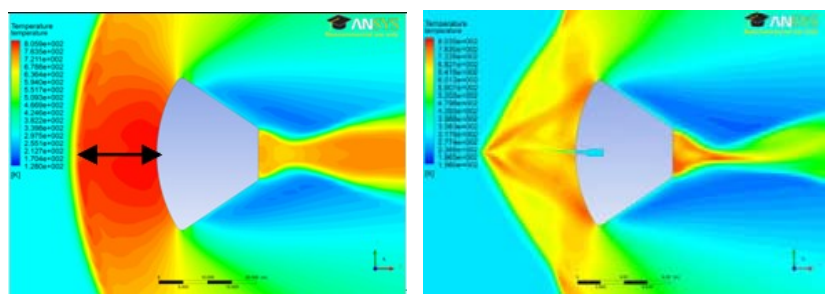

Figure 3: Results with no Jet Simulation Figure 4: Results for Laminar Model

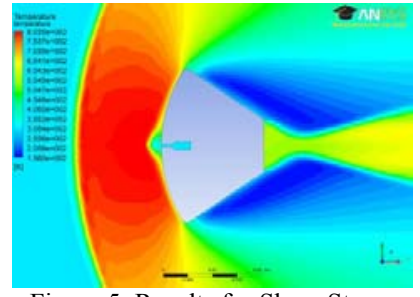

Figure 5: Results for Shear Stress Turbulence Model

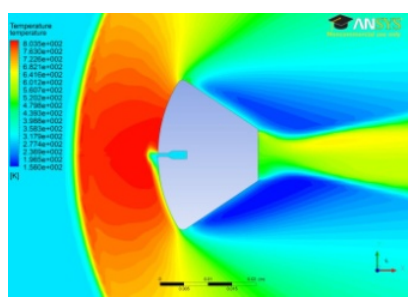

Figure 7: Results for K-Omega Turbulence Model

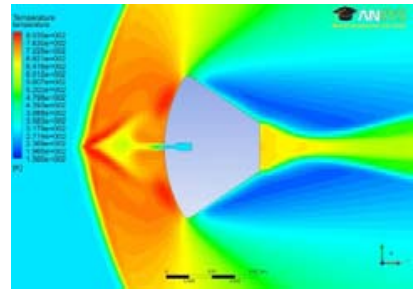

Figure 6: Results for K- $\varepsilon$ Turbulence Model

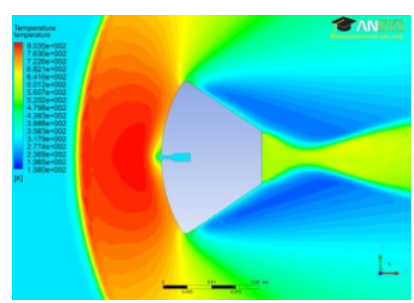

Figure 8: Results for RSL Turbulence Model

From these cases, the distance between bow shock wave and the Apollo body stagnation point under the four turbulence models used are summarized and compared with the standard case without counterflow jet to obtain a comparative estimate of the high temperature region near the body surface were examined. These are shown in Table.1.

TABLE I. DISTANCE BETWEEN SHOCK WAVE AND STAGNATION POINT USING DIFFERENT TURBULENCE MODELS

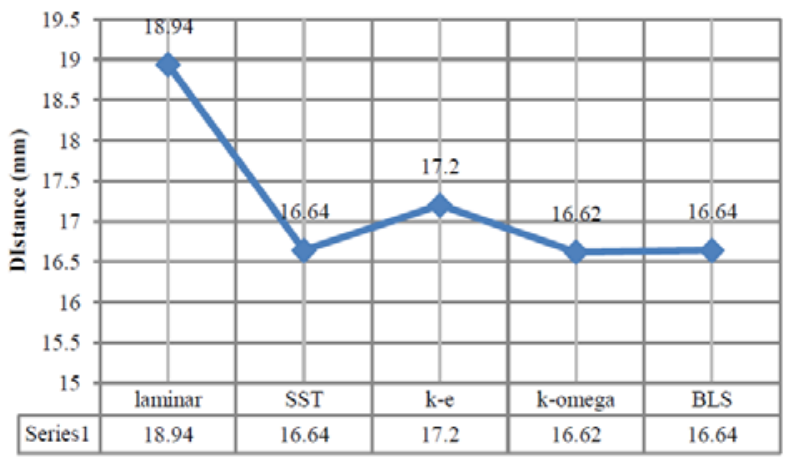

The salient features are highlighted below:

- Laminar model (no turbulence) cannot converge, at Mach 3 as at such high speed turbulence exist.

- Although the $\mathrm{k}-\varepsilon$ model is one of the most common turbulence models, it is not suitable here. K- $\varepsilon$ model has been shown to be useful for free-shear layer flows with relatively small pressure gradients. But this time, the pressure and temperature gradients near body tend to be large. So k- $\varepsilon$ model is not good enough to grasp the whole physical transformation.

- K-Omega model performs significantly better under adverse pressure gradient conditions. Although it is still a bit more time-costing when compared with SST \& BSL Reynolds, it still obtains relatively stable results. But the contour of jet performance seems to be asymmetric

- BSL Reynolds Stress model is Omega-based rather than $\varepsilon$ based for accurate prediction of flow separation. But from the theoretical analysis, BSL model may still fails to properly predict the onset and amount of flow separation from smooth surfaces. The main reason is that this model do not account for the transport of the turbulent shear stress. So when we increase the jet velocity this model may not gives accurate prediction.

- $\quad$ SST model which combines the original Wilcox k-Omega model for use near walls and the standard k- $\varepsilon$ model away from walls using a blending function, and the eddy viscosity formulation is modified to account for the transport effects of the principle turbulent shear stress. It also accounts for the transport of the turbulent shear stress and gives highly accurate predictions of the onset and the amount of flow separation under adverse pressure gradients, therefore SST is the most preferred turbulence model for this case.

\section{CONCLUSIONS}

The present two-dimensional numerical investigation, although limited in scope in terms of quantitative details and accuracy, has been able to provide adequate information of turbulence model usage in numerical simulation and useful 
trends in the early phase of any practical design exercise. This also suggests that for a particular Mach number and geometry, the process of heat dissipation can be improved using counterflow jet ejection and through appropriate optimization can lead to a practical and effective thermal protection system for high speed vehicles. The results also confirm the suitably of using ANSYS as a CFD tool, an important objective of this study.

\section{REFERENCES}

[1] M.Y.M. Ahmed and N. Qin "Recent advances in the aerothermodynamics of spiked hypersonic vehicles," Progress in Aerospace Sciences, vol. 47, pp. 425-449, 2011.

[2] N.A. Ahmed, R.L. Elder, C.P. Foster and J.D.C. Jones, "A Novel 3D Laser Anemometer for Boundary Layer Studies", ASME Conf, Boston, USA, 15th December 1987. Also in the 3rd Int Symp on Laser Anemometry, edited by A Dybs et al, ASME, The Fluids Engg Div, vol 55, pp $175-178$

[3] N.A. Ahmed, R.L. Elder, C.P. Foster and J.D.C. Jones, "Miniature Laser Anem for 3D Meas", Engg Optics, vol 3, No 2, 1990, pp191-196

[4] N.A. Ahmed, R.L. Elder, C.P. Foster and J.D.C. Jones, "Laser Anemometry in Turbomachines", IMechE Proc, Part G, J of Aerospace Engineering, vol 205, 1991, pp1-12

[5] N.A. Ahmed, R. Hamid, R.L. Elder, C.P. Foster and J.D.C. Jones, "Fibre Optic Laser Anemometry for Turbo machinery Applications", Optics and Lasers in Engineering, vol 15, nos 2 and 3, 1992, pp193-205

[6] N.A. Ahmed and R.L. Elder, "Flow Behaviour in a High Speed Centrifugal Impeller Passage under Design and Off-design Operating Conditions", Fluids and Thermal Engineering, JSME International series B, vol.43, No.1 February, 2000, pp22-28

[7] A. Gatto, K.P. Byrne, N.A. Ahmed and R.D. Archer, "Pressure Measurements over a Cylinder in Crossflow using Plastic Tubing", Experiments in Fluids, vol 30, Issue 1, pp 43-46, 2001

[8] N.A. Ahmed, "Implementation of a momentum integral technique for total drag measurement", Iinternational Journal of Mechanical Engineering and Education, vol.30, no.4, 2002

[9] A. Pissasale, and N.A. Ahmed, "Theoretical calibration of a five hole probe for highly three dimensional flow" International Journal of Measurement Science and Technology, vol 13, July, 2002, pp1100-1107

[10] A. Pissasale, and N.A. Ahmed, "A novel method of extending the calibration range of five hole probe for highly three dimensional flows", Journal of Flow Measurement and Instrumentation, vol. 13, issues 1-2, March-April, 2002, pp 23-30

[11] A. Pissasale, and N.A. Ahmed, "Examining the effect of flow reversal on 7-hole probe meas"AIAA Journal, vol 41, no 12, 2003, pp 24602467

[12] A. Pissasale, and N.A. Ahmed, "Development of a functional relationship between port pressures and flow properties for the calibration and application of multi-hole probes to highly 3D flows", Experiments in Fluids, March, vol 36, no.3, March, 2004, pp 422-436

[13] J. Lien and N.A. Ahmed, "An examination of the suitability of multihole pressure probe technique for skin friction measurement in turbulent flow", J of Flow Meas and Instrum, vol. 22, 2011, pp. 153-164

[14] N.A. Ahmed, "Detection of Separation bubble using spectral analysis of fluctuating surface pressure", International Review of Aerospace Engineering', vol.4, no. 4, June, 2011

[15] N.A. Ahmed, "An acoustic energy concept for the design of a flow meter", Int J of Vibration and Acoustics, vol.8, no.1, 2003, pp52-58

[16] R.G. Simpson, N.A. Ahmed and R.D. Archer, "Improvement of a Wing Performance using Coanda Tip Jets", AIAA Journal of Aircraft, vol 37, no 1,2000 , pp183-184 J.

[17] J. Yen and N.A. Ahmed, 'Parametric study of dynamic stall flow field with synthetic jet actuation', Journal of Fluids Engineering, Transactions of the ASME, Volume 134, Issue 7, 2012, Article number 071106
[18] Y.Y Zheng, N.A. Ahmed and W.Zhang, 'Impact Analysis of Varying Strength CounCFJE on a Blunt Shaped Body in A Supersonic Flow', (in press) Advances and Applications in Fluid Mechanics

[19] N.A. Ahmed and R.D. Archer, "Performance Improvement of a Biplane with Endplates", AIAA Journal of Aircraft, vol.38, no.2, pp 398400, March-April, 2001

[20] N.A. Ahmed and R.D. Archer, "Post-Stall Behaviour of A Wing under Externally Imposed Sound", AIAA Journal of Aircraft, vol 38, no.5, pp961-963, September-October, 2001

[21] R.G. Simpson, N.A. Ahmed and R.D. Archer, "Near Field Study of Vortex Attenuation using Wing Tip Blowing", The Aeronautical Journal, vol. 102, March, 2002

[22] N.A. Ahmed and J. Goonaratne, "Lift augmentation of a low aspect ratio thick wing at a very low angle of incidence operating in ground effect", AIAA Journal of Aircraft, vol. 39, no.2, March-April 2002

[23] M. Longmuir and N.A. Ahmed, "Commercial Aircraft Exterior Cleaning Optimization", AIAA, Journal of Aircraft, Vol. 46, No. 1, Jan-Feb issue, 2009, pp284-290

[24] C. Wu and N.A. Ahmed, "Numerical Study of Transient Aircraft Cabin Flowfield with Unsteady Air Supply", AIAA Journal of Aircraft, vol 48, no.6, Nov-Dec issue, 2011, pp. 2164-2169.

[25] S. Shun and N.A. Ahmed, "Utilizing wind and solar energy as power sources for a hybrid building ventilation device", Renewable Energy, Volume 33, Issue 6, June 2008, Pages 1392-1397

[26] N. Findanis and N.A. Ahmed, "The interaction of an asymmetrical localised synthetic jet on a side supported sphere", Journal of Fluids and Structures, Vol. 24 Issue 7, 2008 pp. 1006-1020

[27] N.A. Ahmed, R.L. Elder, C.P. Foster, and J.D.C. Jones, "Miniature Laser Anemometer for 3D Measurements", J of Measurement Sc Technol, vol 1, 1990, pp272-276

[28] N.A. Ahmed and D.J. Wagner, "Vortex shedding and transition frequencies associated with flow around a circular cylinder", AIAA Journal, vol. 41, no.3, March, 2003, pp 542-544

[29] N.A. Ahmed, S. Hamid, R.L. Elder, C.P. Foster, J.D.C. Jones and R Tatum, "Fibre Optic Laser Anemometry for Turbo machinery Applications", Optics and Lasers in Engineering, vol 15, nos 2 and 3, 1992, pp193-205

[30] A. Gatto, N.A. Ahmed and R.D. Archer, "Investigation of the Upstream End Effect of the Flow Characteristics of a Yawed Circular Cylinder",The RAeS Aeronautical Journal, vol 104, No.1033, pp 253256, March, 2000, pp 125-128

[31] N.A. Ahmed, and R.D. Archer, "Testing of a Highly Loaded Horizonatal Axis Wind Turbines designed for Optimum Performance", International $\mathrm{J}$ of Renewable Energy, vol 25, issue 4, pp 613-618, January, 2002

[32] K. Hayashi, S. Aso, and Y. Tani, "Num Study of Therm Protect System by Opposing Jet," 43rd AIAA Aerosp Sci Meet and Exhibit, Nevada,I. Oleksandr, O. Semih, and A.R.S Muhammad, "Experimaental and numerical investigation of shock attenuation using supersonic counter flow jets," 38th Fluid Dyn Conf and Exhibit, Seattle, Washington, 2008.

[33] O. Daso and V.E. Prichett, "The dynamic of shock wave dispersion and interactions in supersonic free streams with counter-flowing jets. ," presented at the 45th AIAA Aerospace Sciences Meeting and Exhibit, Reno, Nevada, 2007.

[34] L. Keysar and D. Degan, "Numerical investigation of axisymmetric compressible turbulent jet," AIAA, vol. 2004-1107, 2004.

[35] B.E. Launder and D.B. Spalding, "The num comp of turbulent flows," Comp Methods in Appl Mech and Engg, vol. 3, pp. 269-289, 1974.

[36] V. Yakhot, S.A. Orszag, S. Thangam, T.B. Gatski, and C.G. Speziale, "Development of turbulence models for sheat flows by a double expansion technique," Physics of Fluids, vol. 4, pp. 1510-1520, 1992.

[37] B.E. Launder, G.J. Reece, and W. Rodi, "Progress in the development of a Reynolds-stress turbulent closure," J of Fluid Mechanics, vol. 68, 1975.

[38] T.H. Shih, W.W. Liou, A. Shabbir, Z. Yang, and J. Zhu, "A new k-e eddy viscosity model for high Reynolds number turbulent flows," Computers and Fluids, vol. 24, pp. 227-238, 1995. 